Petra Szatmári

Budapest

\title{
Niederschlag-Verbvarianten und ihre valenzlexikographische Erfassung
}

\author{
DOI: 10.14232/fest.bassola.14
}

\begin{abstract}
Die synchrone Verbvarianz ist außerordentlich reichhaltig. Auch wenn einige Verwendungen im Sprachgebrauch anteilsmäßig in geringer Anzahl vorkommen, sollten sie lexikographisch erfasst werden, weil sich in ihnen Grundeigenschaften sprachlichen Wissens präsentieren (Engelberg 2010). Der vorliegende Beitrag wendet sich Verbvarianten in erster Linie bei dem Niederschlag-Verb regnen zu und geht deren Erfassung in ausgewählten Wörterbüchern nach.
\end{abstract}

\section{Valenz und Variation}

Auch wenn die Verb-Ergänzungen-Konstellationen im mentalen Lexikon abgespeichert sind und als Satzmuster (oder Satzbaupläne) (vgl. Eroms 2000: 315), d.h. als Grundvalenz, gefasst werden, sind diese Konfiguration jederzeit in der aktuellen Kommunikation qualitativ und / oder quantitativ veränderbar, was Welke als „aktuelle prädikative Leistung“ (Welke 2009: 518) beschreibt. Diese sei „dem Ausbalancieren und der kreativen Weiterentwicklung durch die Sprecher / Hörer auf der Grundlage von Analogien zu anderen Konstruktionen, begleitet von konzeptuellen Anpassungen, geschuldet“ (Welke 2009: 521). Infolge wiederholter Anpassung kommt es zu einer dauerhaften Bedeutungsveränderung, so dass sich die neue Bedeutung neben der ursprünglichen etablieren kann, was dazu führt, dass sie im Wörterbuch erfasst wird.

Das Prädikat, der verbale Valenzträger, „entwirft (kontext- und situationsbezogen) das Szenario, d.h. den einzelsprachlichen Sachverhalt, auf den alle anderen Satzglieder Bezug nehmen“ (Ágel 2017: 255), wodurch Anzahl und syntaktisch-semantische Merkmale der Ergänzungen festgelegt sind. Bedeutsam erscheinen mir in diesem Zusammenhang außerdem die Überlegungen von 
Schöfer (1989), der stark und schwach präsupponierte Ergänzungen annimmt. Während stark präsupponierte zur Perspektivierung der prototypischen Verwendungsvariante gehören und in der Grundvalenz aufscheinen, ist dies bei schwach präsupponierten Ergänzungen nicht der Fall:

Sie können jedoch zusätzlich perspektiviert werden und bewirken damit eine Valenzänderung gegenüber der Grundvalenz. [...] Daneben liefert das Lexikon Informationen über mögliche ,Regelverstöße'. Diese Informationen sind Informationen über mögliche Perspektiveänderungen durch Analogieschlüsse zu anderen Verbmodellen. (Schöfer 1989: 84)

Nach Ágel / Fischer (2010: 270) können mithilfe des Weltwissens, der lexikalischen und syntaktischen Regeln zur Valenzänderung sowie der Kasushierarchie Valenzpotenzial und Valenzrealisierungen aus der inhärenten Lexembedeutung abgeleitet werden. Bleibt trotz der Valenzänderungen das Szenario der inhärenten Lexembedeutung erhalten, sprechen sie von szenarioerhaltenden Valenzänderungen, anderenfalls liegt eine szenarioverändernde Valenzänderung vor.

\section{Synchrone Variierbarkeit der Valenz ausgewählter Niederschlag-Verben}

Die semantisch definierte Verbsubklasse der Witterungsverben lässt sich in Niederschlag-, Gewitter-Verben und Verben der Luftbewegung unterteilen (vgl. Kolehmainen 2008a), denen u.a. folgende Verben zugeordnet werden können:

\begin{tabular}{|c|c|c|}
\hline Niederschlag-Verben & Gewitter-Verben & $\begin{array}{c}\text { Verben der } \\
\text { Luftbewegung }\end{array}$ \\
\hline $\begin{array}{l}\text { es graupelt, es hagelt, es } \\
\text { nieselt, es regnet, es reift, } \\
\text { es schneit, es taut }\end{array}$ & $\begin{array}{l}\text { es blitzt, es donnert, es } \\
\text { klart auf, es gewittert, es } \\
\text { wettert, es wetterleuchtet, }\end{array}$ & $\begin{array}{l}\text { es föhnt, es stürmt, es weht, } \\
\text { es windet, }\end{array}$ \\
\hline
\end{tabular}

Tab. 1: Subklassifikation der Witterungsverben 
Gemeinsam ist ihnen das es-Subjekt, das jedoch nicht die einzige Ergänzung ist. Die im Deutschen metaphorisch bedingte Valenzerhöhung erlaubt es, Witterungsverben u.a. als visuelle Wahrnehmungsverben (Die Augen blitzten vor Erregung. Internetrecherche), Bewegungsverben (Ferres sagte: „Ich donnerte ins Wasser, knallte mit voller Wucht auf ein Metallteil." ntv.de 27.08.2003; Konfetti schneite auf den Karnevalszug, dwds.de) oder Verba dicendi (er donnerte ihr seine Flüche ins Gesicht, duden.de) zu interpretieren.

In Szatmári (im Dr.) bin ich der Variierbarkeit der Verbvalenz ausgewählter Witterungsverben, vornehmlich der des Verbs regnen, nachgegangen. Zur Erfassung der synchronen Valenzvariation von regnen wurden Belege aus den Korpora des Digitalen Wörterbuchs der deutschen Sprache (dwds.de), dem Wortschatzportal der Universität Leipzig (wortschatz.de) sowie der Fachliteratur entnommene Beispiele herangezogen, ergänzt wurden sie durch eigene (Internet-)Recherchen. Die exzerpierten Belege erlauben eine Grobklassifizierung in zwei Subklassen aufgrund der Verbbedeutung: Für die primäre Valenz ist die Bedeutung 'Regen fällt zur Erde' (REgen-Regnen-Szenario) anzusetzen, die mit folgenden Satzmustern verbunden ist:

(a) es-Subj. + Witterungsverb $(\mathrm{WV})_{\text {intransitiv }}$ Es regnet.

(b) es-Subj. $+\mathrm{WV}_{\text {intransitiv }}-$ Direktivum (Dir) ${ }_{(\text {auf/in...) }}$ : Es regnet durch die Decke.

(c) es-Subj. $+\mathrm{WV}_{\text {intransitiv }}-$ Pertinenzdativ $-\mathrm{Dir}_{(\text {auffin...) }}$ : Es regnete ihm ganz bös' ins Gesicht. (https://horb.albverein.eu/schurkenturm/ [03.11.2018])

(d) es-Subj. $+\mathrm{WV}_{\text {transitiv }}-\mathrm{E}_{\text {akk }}$ : Es regnet dicke Tropfen. (Nikula 2006: 915)

Da das Szenario der inhärenten Lexembedeutung erhalten bleibt, liegen in obigen Fällen szenarioerhaltende Valenzänderungen vor. In die zweite Subklasse gehören szenarioverändernde Valenzänderungen, durch die das Fallen von Entitäten (Konkreta / Abstrakta) in großer Dichte und / oder Menge thematisiert wird (Dinge-Regnen-Szenario):

(a) es-Subj. $+\mathrm{WV}_{\text {transitiv }}-\mathrm{E}_{\text {akk }}$ : Für Mannheimer Athleten regnete es Gold-Medaillen (Internetrecherche)

(b) es-Subj. $+\mathrm{WV}_{\text {transitiv }}-\mathrm{E}_{\text {akk }}-$ Dir: Am Schluss regnet es für Furtwängler rote Rosen auf die Bühne [...] (Die Zeit online, 14.01.2013, dwds.de) 
(c) referentielles Subj. $+\mathrm{WV}_{\text {intransitiv }}$ :Rote Rosen regnen, Marmor bricht sich mit Stein und Eisen [...]. (Die Zeit, 27.09.2007, Nr. 39, dwds.de)

(d) referentielles Subj. $+\mathrm{WV}_{\text {intransitiv }}$ - Dir: Blütenblätter regneten auf das Brautpaar (Internetrecherche)

(e) referentielles Subj. $+\mathrm{WV}_{\text {transitiv }}$ - (Dativus commodi) $-\mathrm{E}_{\mathrm{akk}}:$ Und dergestalt steigen die beiden jetzt in Janoschs Phantasiefabel ein: „[...]", rief Pomidore, „sehen Sie nur, der Himmel hat uns ein Schiff geregnet. (Die Zeit, 06.06.1986, Nr. 24, dwds.de)

Obzwar aufgrund der großen Variationsfähigkeit der Verben eine deskriptive Vollständigkeit schier unmöglich erscheint und der prozentuale Anteil der kreativen Verwendungsweisen im Vergleich zur primären Lexembedeutung immer sehr niedrig ist (vgl. Foolen 2008, Engelberg 2014), sollten auch diese weniger häufig vorkommenden kreativen Verwendungen lexikographisch erfasst werden: „Ein Wörterbuch, das dies ignoriert und sich auf die Darstellung des Gebrauchshäufigen beschränkt, ist in dem Maße, in dem es seine Aufgabe in der Erfassung der grundlegenden Strukturen des lexikalischen Systems sieht, unvollständig oder - genauer gesagt - falsch“ (Engelberg 2014: 249), denn das „Spektrum zwischen dem Usuellen und dem Kreativen“ (Engelberg 2014: 251) sei respositorisch zu erfassen.

Im Folgenden konzentriere ich mich - in einem ersten Zugang - auf folgende Varianten: (1) es-Subj. + regnet + Akk, (2) referentielles Subj. + regnen und (3) regnen in Direktivkonstruktionen. Aus Platzgründen kann auf weitere Verwendungen (z.B. in Resultativkonstruktionen, der Eimer regnete voll) im Beitrag nicht eingegangen werden.

\section{1 es-Subj. + regnet + Akk}

Bereits die inhärente Lexembedeutung ist der Erweiterung um eine Akkusativergänzung zugänglich (1).

(1) es regnet Blasen, dicke Tropfen; Es «puddlet» wenn es grosse Flocken schneit (https://www.srf.ch/sendungen/schwiiz-und-duetlich/es-puddletwenn-es-grosse-flocken-schneit [04.01.2019]) 
Nikula (2006) differenziert hier zwischen adverbialer Angabe (es regnet Blasen) und innerem Objekt (es regnet dicke Tropfen). Anders sieht es Kolehmainen (2008b: 171), die auch bei Letzterem von einem Objektkasus ausgeht, weil diese Valenzerhöhung typische Patiensmerkmale zeigt. Für sie handelt es sich deshalb um „normale Versprachlichungsprinzipien“, was sie veranlasst, die Kategorie innere Objekte (ebenso innere Subjekte) zu verwerfen.

Die Entitäten, die in der Akkusativergänzung von regnen kodiert sind, können sowohl Konkreta (2), als auch Abstrakta (3) sein. Vergleichbar mit Es regnet Bindfäden. 'es regnet so stark, dass man meint, einen zusammenhängenden Regenfaden zu sehen' wird ausgedrückt, dass diese Entität in großer Menge und Dichte vorkommt:

(2) In China war ohne Zweifel Unheil im Anzuge, wenn es andere Dinge als Wasser regnete, und die altchinesischen Annalen berichten, daß es Lehm, Steine, Sand, Vögel, Insekten, Menschen, Knochen, Quecksilber, Münzen, Seide und Papier geregnet habe. (Gehlen, Arnold (1956): Urmensch und Spätkultur. dwds.de)

(3) Im Abschnitt ,Ruhrbesetzung' regnet es Ausdrücke wie »erpreßt «, »Ausbeutung «, "Ausschreitungen der Besatzungsangehörigen « [...]. (Tucholsky, Kurt (2000[1929]): Das Nachschlagewerk als politische Waffe, dwds.de)

Die Transitivierung eines primär intransitiven Verbs ist - Kolehmainen (2008b) folgend - eine ganz normale Versprachlichungsstrategie, wie sie bei zahlreichen intransitiven Verben nachzuweisen ist, vgl. (4).

(4) fahren - Wenn vier Leute in einem Auto fahren, dann sind vier Leute Auto gefahren, aber nur einer von ihnen hat das Auto gefahren (es sei denn, sie haben sich beim Fahren abgewechselt). (https://www.heise. de/forum/Autos/Artikel-Foren/Ausfahrt-im-VW-Scirocco/Ein-Autogefahren-haben/posting-2153346/show/[12.11.2018])

Der Rezipient dieses Vorgangs kann in einer für-Phrase kodiert aufscheinen (5).

(5) Es sollte weiter Dollar regnen für seine Familie. (Die Zeit, 28.05.1998, Nr. 23, dwds.de) 
Das Regen-Regnen-Szenario weist zudem gewisse Kollokationen (adverbiale Bestimmungen) auf, die die Art und Weise / Intensität des Regnens (6), Ort oder Zeit / Dauer (8) oder die Richtung / Strecke (9) zum Ausdruck bringen, vgl.

(6) Es regnete nun in dichten Fäden, die der Wind zusammenzwirnte, [...]. (Hahn, Ulla (2003): Unscharfe Bilder. dwds.de);

Dadurch kommt es zu Konstruktionen, in denen einerseits das Wie (adverbiale Angabe) und andererseits das Was (Akkusativergänzung) thematisiert wird, vgl. (7).

(7) Es regnet in dichten, schweren Tropfen. vs. Es regnet warme Tropfen. (Wagner, Siegfried (2004[1923]): Erinnerungen. In: Simons, Oliver (Hrsg.): Deutsche Autobiographien 1690-1930. dwds.de)

(8) Es regnet stärker in diesem Gebirge, die ganze Woche, gerade, ununterbrochen...

(9) Daß eine Gegend der Erde existiere, wo [...] die Bäume abwärts wachsen und es in die Höhe regnet, hagelt und schneit? (Fleck, Ludwik (1980[1935]): Entstehung und Entwicklung einer wissenschaftlichen Tatsache. dwds.de); Jeder kennt die Wolken am Himmel und weiß auch, dass sie aus Wasser bestehen und es daher auch aus ihnen regnen kann. (https://content. meteoblue.com/ de/meteoscool/wetter/wolken [14.11.2018])

Durch das Hinzufügen des Direktivums wird das Witterungsverb mit Bewegung verknüpft und kann analog zu den Satzmustern der Bewegungsverben die beiden Endpunkte einer Bewegung (Quelle / Herkunft und Ziel) versprachlichen. Auf Schöfer (1989) referierend sind Akkusativ und Direktivum schwach präsupponierte Ergänzungen. Werden sie von der Sprachgemeinschaft häufig erfolgreich eingesetzt, kann sich dies auf die Veränderung der Grundvalenz auswirken: Direktivkonstruktionen mit zwei und drei Argumenten werden auf Witterungsverben ausgedehnt und lexikalisiert, vgl. Abschnitt 2.3.

\section{2 referentielles Subj. + regnen}

Wie bereits festgestellt, sind referentielle Subjekte in diesen Konstruktionen möglich (10). In erster Linie kommen Entitäten aus dem Bereich der Naturer- 
scheinungen vor (Luft, Himmel), aber auch völlig andere Bereiche werden zur Füllung der Subjektposition genutzt (Film).

(10) Die Luft kann hageln und regnen, dagegen kann man sich nicht wehren, aber gegen vieles andere kann man sich wehren. (Döblin, Alfred (1961[1929]): Berlin Alexanderplatz. dwds.de); So wie der Himmel regnet, so weinen die Engel [...]. (Schwanitz, Dietrich (1999): Bildung. dwds. de); [...] es war ein alter Stummfilm, er flitterte und regnete [...] (Kreuder, Ernst (1978[1946]): Die Gesellschaft vom Dachboden. dwds.de)

\section{3 regnen in Direktivkonstruktionen}

Regnen ist sowohl in Konstruktionen mit es-Subj. (11) als auch in solchen mit referentiellem Subj. (12) mit einem Direktivum kombinierbar.

(11a) Dann kam die Linde, kurzstämmig, mit einer mächtigen, halbkugeligen Krone, [...], und aus der es in klaren, sonnenblitzenden Tropfen regnete. (Frapan, Ilse (2001[1903]): Arbeit. In: Deutsche Literatur von Frauen. dwds.de)

(11b) [...] und schon regnet es verendete Käfer in die aufgespannten Netze der Forscher. (Die Zeit, 23.03.2000, Nr. 13, dwds.de)

(12a) Kiloschwere Eisbrocken regnen vom Himmel, man weiß nicht recht, wie und warum. (Die Zeit, 27.01.2000, Nr. 5, dwds.de)

(12b) Jeden Abend [...] muss er auf das Dach klettern, um Zweige und Dreck zu entfernen, damit Baumaterial und Ausscheidungen der Vögel nicht in den Kamin und auf das Fensterbrett der vermieteten Dachwohnung (von Oma Specht) regnen. (Die Zeit, 06.04.2000, Nr. 15, dwds.de)

(12c) Beeren unbekannter Herkunft regnen durch anthrazitfarbene Luft [...]. (Riedel, Susanne (2003): Eine Frau aus Amerika. dwds.de)

So werden Quelle ((11a), (12a)), Ziel ((11b), (12b)) und Weg (12c) thematisiert. In Analogie zum Gebrauch der Geräuschverben als Bewegungsverben, den Eroms (2012) als reguläre Valenzerhöhung verbucht, sehe ich diese Konstruktionen. ${ }^{1}$

Vor dem Hintergrund, dass Valenzen „idiosynkratische syntaktische Programme“ (Eroms 2012: 32) notieren, meint Eroms, dass es sich bei den direktionalen Präpositionalphrasen in den mit Geräuschverben gebildeten Kompaktkonstruktionen um Ergänzungen handelt. 
Niederschlag-Verben folgen demnach diesem Muster etablierter Versprachlichungsstrategien.

Bei einigen Konstruktionen mit regnen zeigt sich eine weitere Analogie zu Versprachlichungsstrategien: Dieselbe Entität lässt sich im Subjekt bzw. in der Akkusativergänzung kodieren (13). Dies ist häufig bei konkreten NP-Referenten der Fall (Geld / Konfetti / Bomben). Dass abstrakte Referenten eher als Akkusativergänzung realisiert werden (14), führt Kolehmainen (2008a) auf deren geringere Agenshaftigkeit zurück, dennoch kommen auch sie als Subjekt vor (15).

(13) Bei „Wunderbar“ aus Kiss Me, Kate (Cole Porter) regnete goldenes Konfetti von der Decke [...]. (Die Zeit online, 31.12.2015, dwds.de) vs. In wenigen Stunden wird es als Zugabe goldenes Konfetti regnen, [...]. (Die Zeit, 19.03.2015, Nr. 12, dwds.de)

(14) Von nun an regnete es Überraschungen. (Klemperer, Victor (2000 [1926]): [Tagebuch] 1926. In: Ders.: Leben sammeln, nicht fragen wozu und warum. Berlin, 100, dwds.de)

(15) [...] was aber soll er auf die Fragen antworten, die gleich morgen früh auf ihn regnen werden? (Becker, Jurek (1969): Jakob der Lügner. dwds.de)

Mit den Direktivkonstruktionen ist eine weitere Eigentümlichkeit verknüpft. Das Auftreten des es-Subjekts stabilisiert die Perfektbildung mit haben (16), tritt dagegen in Direktivkonstruktionen ein referentielles Subjekt auf, passt sich die Konstruktion den Bewegungsverben an und bildet ihr Perfekt mit sein (17).

(16) Es sieht aus, als hätte es riesige Zuckerwürfel geregnet. (Die Zeit, 23.02.2015 (online), dwds.de)

(17) Gelber Wüstenstaub ist in München und dem Alpenvorland vom Himmel geregnet. (Die Zeit, 20.02.2014 (online), dwds.de)

\section{Allgemeines zu Valenzinformationen in Wörterbüchern}

In Allgemeinwörterbüchern werden in der Regel Valenzinformationen zum Verb gegeben, die der Wörterbuchbenutzer extrahieren muss, um daraus die Regularitäten für den produktiven Gebrauch des Verbs abzuleiten. Bei Lernerwörterbüchern, die als trifunktional zu betrachten sind, weil sie zur ,übersetzerischen 
und nichtübersetzerischen Textrezeption und Textproduktion [sowie zur] Kompetenzerweiterung" genutzt werden (Järventausta 2006: 46), wird diese Kompetenz nicht erwartet, so dass die Darstellungsweise des jeweiligen Lemmas neben morphosyntaktischen, semantischen und pragmatischen Valenzinformationen auch eine verständliche Beschreibungssprache erforderlich macht.

Während sich Muttersprachler die valenziellen Informationen $\mathrm{zu}$ einem Prädikat im Spracherwerb aneignen, muss diese Kompetenz im Fremdsprachenerwerb sukzessive aufgebaut werden. Erschwert wird die Aneignung solcher Kenntnisse dadurch, dass Valenzeigenschaften einerseits innerhalb einer Sprache und andererseits von Sprache zu Sprache variieren, deshalb kommt den Valenzinformationen im Fremdsprachenerwerb bzw. bei der Erstellung von Grammatiken und Wörterbüchern eine grundlegende Bedeutung zu (Järventausta 2006, Engelberg 2010, Hollós 2014).

Indes ist die tradierte Teilung von Grammatikographie und Lexikographie für die Sprachbeschreibung in der heutigen Zeit nicht mehr haltbar. Sie führe nach Engelberg (2010: 114) „zu einer inkonsistenten und wenig reflektierten Beschreibung eines großen Bereichs von Phänomenen“. Er bezieht sich damit auf Strukturen (bei Engelberg „konstruktionelle Varianten“), die Argumentrealisierungen aufweisen, die im Standardlexikoneintrag / in der Grundvalenz des Verbs nicht vorgesehen sind (vgl. Engelberg 2010: 114). Derartige sprachliche Phänomene werden zwar relativ selten realisiert, dennoch meint Engelberg (2014), dass darin Grundeigenschaften sprachlichen Wissens ihren Ausdruck finden würden, indem „das Häufige dem kognitiv einfach zu Verarbeitenden, Unauffälligen entspricht, und das Seltene dem kognitiv Aufwändigen, dem Auffälligen und Kreativen“ (Engelberg 2014: 249). In der „Darstellung des Spannungsbogens zwischen dem Üblichen und dem Kreativen“ sieht er u.a. das Leistungsvermögen zukünftiger sprachdokumentarischer Internetwörterbücher. ${ }^{2}$

Eine verwendungsorientierende Funktion haben Beispielsätze in Grammatik- bzw. Wörterbüchern. Nikula stellt fest, dass es sich dabei stets um „ein aus dem Zusammenhang gerissenes Zitat“ (Nikula 1988: 484, auf Hermanns (1988) referierend) handelt, das innerhalb des metasprachlichen Kontextes

\footnotetext{
2 Die Bedeutung der Internetlexikographie bestätigt eine Erhebung von Dringó-Horváth (2017), nach der etwa 50\% der Befragten auf Online-Wörterbücher zurückgriffen, um Informationen in Bezug auf Aussprache, Schreibweise, Grammatik oder für Übersetzungszwecke einzuholen.
} 
eine zweifache Aufgabe erfüllt, indem es einerseits der Verständlichmachung der Regelformulierung und andererseits der Angabe der Sprachregeln dient und damit „als grammatisches oder lexikographisches Beispiel [...], genau wie das konstruierte Beispiel oder ,Kompetenzbeispiel', eine Modellfunktion [hat]“ (Nikula 1988: 484). Diese Belegbeispiele können in jedem Fall vom Benutzer / von der Benutzerin der Grammatik oder des Wörterbuchs als Modell für eigene Bildungen eingesetzt werden. Bei zweisprachigen Wörterbüchern erweist sich - wie Bassola (2006) feststellt - ihre Beschreibung mithilfe von grammatischen Termini oder Proformen, die dann in den Beispielen optisch hervorgehoben werden, als außerordentlich hilfreich.

\subsection{Zur lexikographischen Erfassung des Verbs regnen}

Zunächst seien die Satzmuster des Verbs regnen in häufig konsultierten einsprachigen Printwörterbüchern - für Vergleichszwecke leicht abgewandelt wiedergegeben:

\begin{tabular}{|c|c|c|}
\hline Wahrig (1991) & VALBU (2004) & LGDaF (2015) \\
\hline $\begin{array}{l}\text { regnen <V. i.; unper- } \\
\text { sönl.; umg. u. fig. a. } \\
\text { V. t.> sich als Tropfen } \\
\text { aus den Wolken nie- } \\
\text { derschlagen; <fig.> in } \\
\text { Mengen kommen, ein- } \\
\text { treffen, zuströmen; es } \\
\text { regnet es fällt Regen; es } \\
\text { regnete Anfragen, Be- } \\
\text { schwerden, Glückwün- } \\
\text { sche, Vorwürfe <fig.; } \\
\text { umg.>; es regnet Bind- } \\
\text { fäden, Schusterjungen } \\
<\text { fig.; umg.; scherzh.> } \\
\text { es regnet heftig; fein, } \\
\text { stark ; es regnet in } \\
\text { Strömen [<ahd. regan- } \\
\text { non; } \rightarrow \text { Regen>] }\end{array}$ & $\begin{array}{l}\text { regnen, es es regnet - es regne- } \\
\text { te - es hat geregnet } \\
\mathbf{1} \text { Regen fällt: Plötzlich fing es } \\
\text { an zu regnen. } \\
2 \text { AkkE es fällt etwas [konkr. } \\
\text { Objekt: Konfetti o.Ä./Substanz: } \\
\text { Asche, Sand o.Ä.] in großer Men- } \\
\text { ge herab: [Vor einigen Jahren] } \\
\text { regnete es gelben Wüstensand. } \\
3 \text { AkkE es gibt eine große An- } \\
\text { zahl von etwas [abstr. Objekt/ } \\
\text { Handlung: Anruf, Glück- } \\
\text { wunsch o.Ä.] [Beim 60. Ge- } \\
\text { burtstag von unserem Direk- } \\
\text { tor] hat es Glückwünsche und } \\
\text { Ehrungen geregnet. } \\
4 \text { AdvE (auf + A/in + A/...) Re- } \\
\text { gen fällt irgendwohin } \\
\text { Es regnete [mir] in den Kragen. } \\
\text { - Pertinenzdativ ist möglich }\end{array}$ & $\begin{array}{l}\text { regnen }_{\mathrm{V} / \mathrm{IMP}}<\text { regnete, hat } \\
\text { geregnet }>1 \text { es regnet es } \\
\text { fällt Regen zur Erde <es } \\
\text { regnet leicht, stark, heftig, } \\
\text { in Strömen }>2 \text { es regnet } \\
\text { Dinge etwas fällt in großen } \\
\text { Mengen herunter | Im Kar- } \\
\text { neval regnet es Konfetti } 3 \text { es } \\
\text { regnet Dinge gesprochen } \\
\text { jemand bekommt etwas in } \\
\text { großen Mengen | Es regnete } \\
\text { Anfragen }\end{array}$ \\
\hline
\end{tabular}




\begin{tabular}{|l|l|l|}
\hline „Nicht behandelt wird hier das & V/IMP = unpersönlich ge- \\
Verb regnen“ (VALBU 2004: & brauchtes Verb (mit es als \\
602 ); außerdem wird darauf & Subjekt); gesprochen = Wör- \\
verwiesen, dass kein Passiv ge- & ter oder Wortverbindungen, \\
bildet werden kann. & $\begin{array}{l}\text { die „- normalerweise - nur } \\
\text { in der gesprochenen Spra- } \\
\text { che“ (LGDaF 2015: 19) Ver- } \\
\end{array}$ \\
& wendung finden \\
\hline
\end{tabular}

Tab. 2: Lemma regnen in ausgewählten Printwörterbüchern

Indem authentische Beispiele im VALBU-Wortartikel angeführt werden, ist dieser am längsten. Darin werden nicht obligatorische Ergänzungen / Argumente (durch runde Klammern) und Angaben (durch eckige Klammern) kennzeichnen, was bei den anderen beiden Nachschlagewerken entfällt. Gemeinsam ist den Wörterbüchern somit die Kennzeichnung obligatorischer Mitspieler, so dass dem Lerner bei der Sprachproduktion durchaus Hilfe geboten wird. Allerding stellt eine echte Erleichterung für DaF-Lerner die Benennung der Ergänzungsklasse im VALBU dar. In den beiden anderen Wörterbüchern muss der Benutzer sich die Ergänzungsklasse erschließen. Auf die Möglichkeit des Direktivums und Pertinenzdativs verweist einzig das VALBU.

Nicht unproblematisch ist, dass nur die unpersönliche Verwendung aufgenommen wurde, lediglich das VALBU vermerkt, dass es „noch“ ein Verb regnen gibt.

Im Gegensatz dazu nimmt das häufig konsultierte Internetwörterbuch duden. de keine derartige Trennung vor. $\mathrm{Zu}$ den untersuchten Stichwörtern finden sich dort folgende Informationen (aus Gründen der Übersicht wurden sie formal verändert): ${ }^{3}$

Bedeutung, Grammatik: regnen schwaches Verb

(1) (von Niederschlag) es regnet leise, sanft, stark, heftig, ununterbrochen, tagelals Regen zur Erde fallen; unpersönang, in Strömen lich; Perfektbiles fängt an, hört auf zu regnen dung mit »hat» es regnet an die Scheiben, aufs Dach es regnete große Tropfen (der Regen fiel in großen Tropfen)

\footnotetext{
3 Das Internetwörterbuch duden.de gibt darüber hinaus Informationen zur Häufigkeit, Rechtschreibung, Aussprache und Herkunft, listet Synonyme auf und ordnet das Wort im Wortschatz des Goethe-Zertifikats ein.
} 


\begin{tabular}{|l|l|}
\hline (2) in großer Menge, & von den Rängen regneten Blumen \\
wie ein Regen nie- & $<$ in übertragener Bedeutung>: Vorwürfe, Schimpfworte \\
dergehen, herabfal- & regneten auf ihn (bekam er in großer Menge zu hören) \\
len; Perfektbildung & $<$ in übertragener Bedeutung>: nach der Fernsehübertra- \\
mit »ist « & $\begin{array}{l}\text { gung regnete es bei dem Sender Beschwerden }(\text { Beschwer- } \\
\text { den gingen in großer Zahl bei dem Sender ein })^{4}\end{array}$ \\
\hline
\end{tabular}

Tab. 3: Lemma regnen im Internetwörterbuch duden.de

Duden.de erfasst sowohl das Regen-Regnen- als auch das Dinge-Regnen-Szenario. In Letzterem werden Abstrakta mit dem Vermerk „in übertragener Bedeutung" versehen und nur zu diesen wird die Variante mit dem es-Subj. angeführt. $\mathrm{Da}$ laut Fachliteratur vor allem Konkreta sowohl in Subjekt- als auch $\mathrm{E}_{\text {akk }}$-Position vorkommen, werden hier dem Lernenden wesentliche Informationen vorenthalten. Das Internetwörterbuch nutzt außerdem auch andere Möglichkeiten der Markierung nicht. Ich denke hier u.a. an die Benennung der Ergänzungsklassen und ihre farbliche Kennzeichnung im Beispiel. Dadurch wird der kognitive Prozess, in dem sich der Wörterbuchbenutzer befindet, zusätzlich unterbrochen, denn er muss zunächst die notwendigen Regularitäten extrahieren.

Besonders wichtig für Nicht-Muttersprachler sind die Hinweise zur Perfektbildung. Da die diesbezüglichen Angaben nicht eindeutig sind, führen sie bei ungeübten Benutzern eher zu Verwirrungen.

\section{Fazit}

Aus den Wörterbuchartikeln wird ersichtlich, dass bei obigem Niederschlag-Verb die Verwendung mit $\mathrm{E}_{\mathrm{akk}}$ Aufnahme gefunden hat, ohne dass jedoch die Verwendungsvariationen in ihrer gesamten Breite erfasst werden (Direktivum, Pertinenzdativ).

Konstruktionen mit referentiellem Subj. + Dir, bei denen es sich zwar um lexikalisierte Verwendungen handelt, haben noch nicht den Weg in jedes Wörterbuch gefunden.

\footnotetext{
4 Bei dieser Verwendungsweise wird nicht darauf hingewiesen, dass die Perfektbildung mit haben erfolgt.
} 
Generell zeigt sich, dass besonders Nicht-Muttersprachler in nicht ausreichendem Maße Hilfe durch die Wörterbücher bekommen, da sie sich in den meisten Fällen die Regularitäten für die produktive Verwendung aus den Beispielen ableiten müssen.

\section{Literatur}

\subsection{Primärliteratur / Quellen}

LGDaF = Götz, Dieter (Hrsg.) (2015): Langenscheidt Großwörterbuch Deutsch als Fremdsprache. München / Wien: Langenscheidt.

VALBU $=$ Schumacher, Helmut / Kubczak, Jacqueline / Schmidt, Renate / de Ruiter, Vera (2004): VALBU - Valenzwörterbuch deutscher Verben. Tübingen: Narr.

Wahrig, Gerhard (1991): Deutsches Wörterbuch. Gütersloh / München: Bertelsmann.

\subsection{Sekundärliteratur}

Ágel, Vilmos (2017): Grammatische Textanalyse. Textglieder, Satzglieder, Wortgruppenglieder. Berlin / Boston: Walter de Gruyter.

Ágel, Vilmos / Fischer, Klaus (2010): 50 Jahre Valenztheorie und Dependenzgrammatik. In: Zeitschrift für germanistische Linguistik 38, 249-290.

Bassola, Peter (2006): Valenzinformationen in allgemeinen zweisprachigen Wörterbüchern. In: Ágel, Vilmos et al. (Hrsg.): Dependenz und Valenz. Ein internationales Handbuch der zeitgenössischen Forschung. Berlin / New York: Walter de Gruyter (HSK 25.2), 1387-1396.

Dringó-Horváth, Ida (2017): Digitális szótárak - szótárdidaktika és szótárhasználati szokások. In: Alkalmazott Nyelvtudomány, Különkiadás 2017, 1-27. http://alkalmazottnyelvtudomany.hu/ wordpress/wp-content/uploads/ DringoHorvath.pdf (gesichtet am 16.12.2018).

Engelberg, Stefan (2010). Die lexikographische Behandlung von Argumentstrukturvarianten in Valenz- und Lernerwörterbüchern. In: Schierholz, Stefan J. / Fobbe, Eilika / Fischer, Klaus (Hrsg.): Valenz und Deutsch als Fremdsprache: Frankfurt a.M.: Lang, 113-141. 
Engelberg, Stefan (2014): Gegenwart und Zukunft der Abteilung Lexik am IDS: Plädoyer für eine Lexikographie der Sprachdynamik. In: Steinle, Melanie / Berens, Franz Josef (Hrsg.): Ansichten und Einsichten. 50 Jahre Institut für Deutsche Sprache. Mannheim: Institut für Deutsche Sprache, 243-253.

Eroms, Hans-Werner (2000): Syntax der deutschen Sprache. Berlin / New York: Walter de Gruyter.

Eroms, Hans-Werner (2012): Die Grenzen der Valenzen. In: Fischer, Klaus / Mollica, Fabio (Hrsg.): Valenz, Konstruktion und Deutsch als Fremdsprache. Frankfurt a.M.: Lang, 25-46.

Foolen, Ad (2008): Verbale Flexibilität: Zwischen Valenz und Konstruktion. In: Foolen, Ad / van Gemert, Guillaume (Hrsg.): Deutsch und Niederländisch in Sprache und Kultur. Aufsätze für Jan van Megen zu seinem Abschied von der Radboud Universiteit Nijmegen. Nijmegen: Tandem Felix, 1-11.

Hermanns, Fritz (1988): Das lexikographische Beispiel. Ein Beitrag zu seiner Theorie. In: Harras, Gisela (Hrsg.): Das Wörterbuch. Artikel und Verweisstrukturen. Jahrbuch 1987 des Instituts für deutsche Sprache. Düsseldorf: Schwann (Sprache der Gegenwart 74), 161-195.

Hollós, Zita (2014): Syntagmatik im KOLLEX. Die lexikographische Darstellung grammatischer Syntagmatik in einem zweisprachigen Kollokationslexikon für Deutschlerner. In: Domínguez Vázquez, María José / Mollica, Fabio / Nied Curcio, Martina (Hrsg.): Zweisprachige Lexikographie zwischen Translation und Didaktik. Berlin / Boston: Walter de Gruyter (Lexikographica 147), 113-129.

Järventausta, Marja (2006): Valenzielle und konstruktionelle Informationen in einem lernerorientierten Verbwörterbuch. In: Neuphilologische Mitteilungen 107.1, 45-85.

Kolehmainen, Leena (2008a): Es schneit wattebauschgroße Schneeflocken: Valenz, Proto-Rollen und Argumentstruktur-Konstruktionen bei deutschen und finnischen Witterungsverben. In: Enell-Nilsson, Mona / Männikkö, Tiina (Hrsg.): Erikoiskielet, käännösteoria ja monikielisyys, VAKKI-symposiumi XXVIII. Vaasa 8.-9.2.2008. Vaasa, 100-112. http://www.vakki.net/ publications /no35_fin.html (gesichtet am 06.01.2019).

Kolehmainen, Leena (2008b): Valenzalternationen im zwischensprachlichen Vergleich, die sog. inneren Objekte im Deutschen und Finnischen. In: Neuphilologische Mitteilungen 109.2, 155-178. 
Nikula, Henrik (1988): Zur Frage der Textualität von Beispielsätzen. In: Neuphilologische Mitteilungen 89.4, 483-488.

Nikula, Henrik (2006): Unpersönliche Konstruktionen. In: Ágel, Vilmos et al. (Hrsg.): Dependenz und Valenz: Ein internationales Handbuch der zeitgenössischen Forschung. Berlin / New York: Walter de Gruyter (HSK 25.2), 913-920.

Schöfer, Göran (1989): Prinzipien der Valenzänderung: Am Beispiel des deutschen Dativs. In: Zeitschrift für Germanistik 10.1., 83-90.

Szatmári, Petra (im Dr.): Es regnete Anfragen und ehe er sich`s versah, schneite ihm eine Überraschung ins Haus. Zur synchronen Variierbarkeit der Valenz der Witterungsverben. In: Błachut, Edyta / Gołębiowski, Adam (Hrsg.): Markiertheit, Markers. Phänomene im syntaktischen, semantischen und pragmatischen Bereich.

Welke, Klaus (2009): Konstruktionsvererbung, Valenzvererbung und die Reichweite von Konstruktionen. In: Zeitschrift für germanistische Linguistik 37, 514-543. 